\title{
FNCLCC Sarcoma Grade 2
}

National Cancer Institute

\section{Source}

National Cancer Institute. FNCLCC Sarcoma Grade 2. NCI Thesaurus. Code C9028.

A sarcoma with a total score of 4 or 5 according to the FNCLCC guidelines. 